\title{
Chapter 4 \\ Behavioral Exchange and Interchange as Strategies to Facilitate Social Relationships in Tibetan Macaques
}

\author{
Dong-Po Xia, Paul A. Garber, Cédric Sueur, and Jin-Hua Li
}

\subsection{Introduction}

Since the 1970s, sociobiologists have focused on two issues when studying social animals. One is the evolutionary processes that have favored group living, and the other is how ecological factors influence the internal structure of groups and the nature of social relationships and how changes in behavior influence the costs and benefits of group living to individuals of different age, sex, and dominance status (Wilson 1975; Barash 1977; Morse 1980; Wrangham 1980; Krebs and Davies 1984; van Schaik 1989). Studies on social animals have argued that the benefits of group living (such as social learning, cooperative hunting, collective resource and mate defense, cooperative infant caregiving, increased foraging efficiency, a reduction in stress associated with social support and reconciliatory behavior, alliance formation,

D.-P. Xia

School of Life Sciences, Anhui University, Hefei, China

International Collaborative Research Center for Huangshan Biodiversity and Tibetan Macaque Behavioral Ecology, Anhui, China

P. A. Garber

Department of Anthropology, Program in Ecology, Evolution, and Conservation Biology,

University of Illinois, Urbana, IL, USA

e-mail: p-garber@illinois.edu

C. Sueur

CNRS, IPHC, UMR, Université de Strasbourg, Strasbourg, France

e-mail: cedric.sueur@iphc.cnrs.fr

J.-H. Li (ه)

School of Resources and Environmental Engineering, Anhui University, Hefei, Anhui, China

International Collaborative Research Center for Huangshan Biodiversity and Tibetan Macaque Behavioral Ecology, Anhui, China

School of Life Sciences, Hefei Normal University, Hefei, Anhui, China

e-mail: jhli@ahu.edu.cn 
and partner reliability) serve to counteract the costs and offer fitness benefits to individuals living in a well-functioning and stable social unit (e.g., multilevel selection, Krause and Ruxton 2002; Sussman and Garber 2011).

According to Hinde (1976), social structures are group characteristics based on the patterns of social relationships such as affiliative, cooperative, sexual, and agonistic interactions that characterize a species or members of an established social unit. In order to maintain group cohesion, individuals are required to coordinate their daily activities, including collective movement to feeding and resting sites. Understanding how this is accomplished, whether individuals of a particular age, sex, or rank class consistently lead or direct group movement, and how "leaders" build a consensus in deciding when to leave a feeding or resting site and which location to travel to next, offers critical insights into the dynamics of animal social systems (see also Chap. 5).

A recent paper by Schino and Aureli (2017) describes two alternative processes associated with social relationships. Partner control highlights dyadic interactions between dyads in which the behavior of each subject is dependent exclusively on the previous behavior of a partner (Noë 2006). This assumes that one member of a dyad can control the behavior of its partner with no opportunity of partner switching. Partner control has been used to describe social interactions between individuals living in a social unit. Partner choice assumes that individuals can freely select and change partner preferences based on the assessed benefit each potential partner is expected to provide. For example, in studies of Sapajus nigritus (formerly Cebus apella) and Macaca fuscata (Schino and Aureli 2009, 2017; Schino et al. 2007, 2009), partner choice was found to influence social relationships and group stability.

Biological market theory proposes that social behaviors can be considered as valuable commodities and explains how commodities are exchanged or interchanged among group members (Noë and Hammerstein 1994, 1995; Noë 2006). This theory considers social behaviors as exchangeable commodities to obtain behavioral services as benefits (Noë 2001; Barrett and Henzi 2006; Noë \& Voelkl 2013). Individuals compete over access to partners, by offering more valuable services rather than engaging in aggressive or coercive behavior. Therefore, individuals are chosen as partners depending on the services they offer, and the choice is made by comparing the offers of all potential partners depending on the services needed. As such, biological market theory provides an alternative framework to study social relationships and partner preferences and facilitates the understanding of behavioral adaptations for group stability and social cohesiveness in group-living animals.

In most nonhuman primates, behavioral exchange and interchange have been well documented (reviewed in Sánchez-Amaro and Amici 2015). For example, Seyfarth (1977) proposed that lower-ranking females compete to groom higher-ranking females who can offer them more effective aid (agonistic support) in competitive interactions with other group members. In some species such as chacma baboons, Papio cynocephalus (Barrett et al. 1999); red-fronted lemurs, Eulemur fulvus rufus (Port et al. 2009); and chimpanzees, Pan troglodytes (Newton-Fisher and Lee 2013; Kaburu and Newton-Fisher 2015a), grooming is reported to be exchanged for grooming (reciprocal exchange). Within a dyad consisting of individuals A and B, 
the amount of grooming given by A to B depends on the amount of grooming given by $\mathrm{B}$ to $\mathrm{A}$. In some other species, studies show that behavioral services can also be interchanged for different behaviors. For example, grooming can be interchanged for food sharing to maintain social bonds and agonistic support in chimpanzees (de Waal 1997; Kaburu and Newton-Fisher 2015a). In sooty mangabeys (Cercocebus atys) and vervet monkeys (Chlorocebus aethiops), infant handling can influence dyadic relationships. In this species, grooming is interchanged for opportunities to handle infants in order to establish social relationships (Fruteau et al. 2011). In long-tailed macaques (M. fascicularis), grooming is interchanged for tolerance to increase spatial proximity and to reduce aggression from higher-ranking individuals (Gumert and Ho 2008). Additionally, grooming can be interchanged for copulations (Kaburu and Newton-Fisher 2015b). Mating opportunities increase for females who groom a higher-ranking male compared to females who groom a lowerranking male (Gumert 2007a). Similarly, Gomes and Boesch (2009) found that over a 22-month period, female chimpanzees copulated more with males who shared meat with them compared to males who did not engage in food sharing. In this species, food sharing by adult males provides more mating opportunities.

In this chapter, our goal is to review the evidence for multiple behavioral exchanges and interchanges in Tibetan macaque (see Chap. 2 for more information about Tibetan macaques) groups at Mt. Huangshan, China (Fig. 4.1), and to analyze these dyadic behavioral interactions within the context of behavioral exchange or interchange. We define exchange as the reciprocation of the same behaviors (e.g., grooming for grooming) and interchange as the reciprocation between different

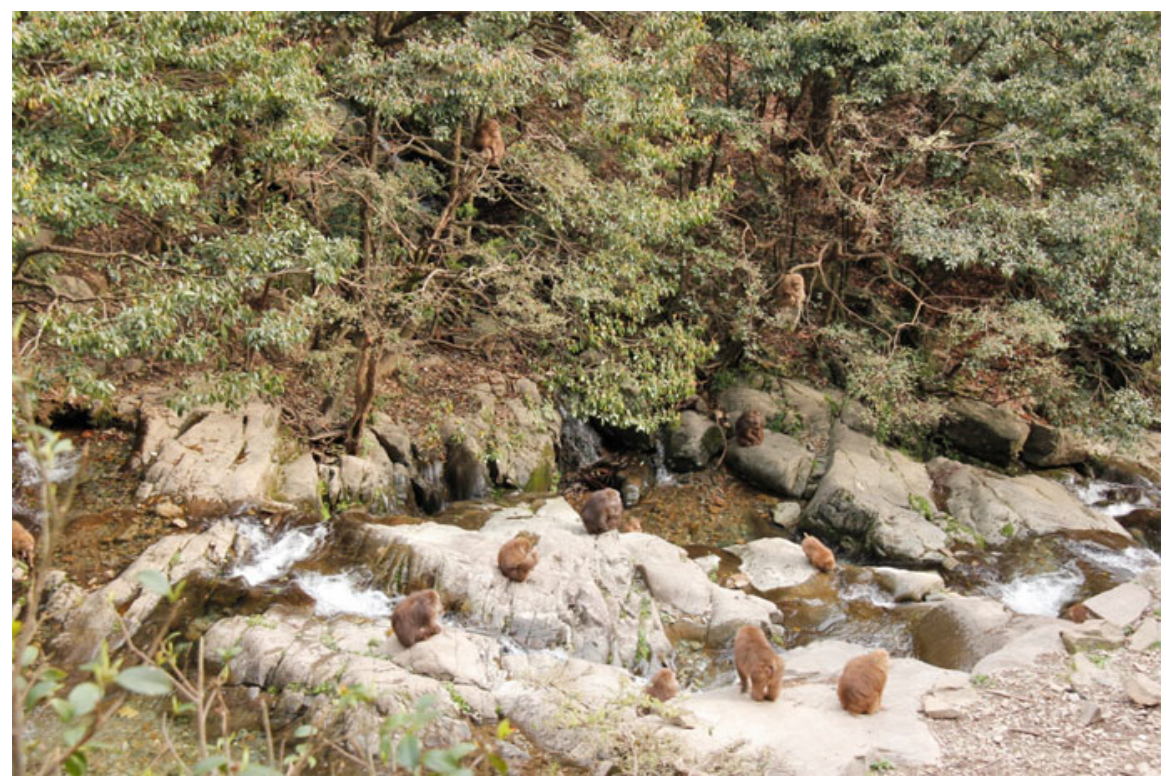

Fig. 4.1 A social group (known as YA1) of Tibetan macaques at Mt. Huangshan, China 
behaviors of the same category (e.g., grooming for tolerance, which are different behaviors but both belong to the same category of affiliative relationships) or behaviors of different categories (e.g., agonistic support for copulation). We focus on grooming exchanges, the interchange of grooming for tolerance among female or male intrasexual dyads, the interchange of grooming for infant-handling opportunities among females, and the interchange of male-to-female agonistic support for mating opportunities among intersexual dyads.

\subsection{Exchange Between the Same Behaviors}

\subsubsection{Grooming for Itself}

Grooming has been reported to be exchanged for grooming (reciprocal exchange) in primates (such as chacma baboons, Barrett et al. 1999). In Tibetan macaques, similarly, approximately $20 \%$ of their daily activity budget is devoted to grooming, regardless of sex, social rank, age, and other factors (see Xia et al. 2012, 2013) (Fig. 4.2 shows grooming in Tibetan macaques). Xia et al. (2012, 2013) examined female and male intrasexual grooming relationships in free-ranging Tibetan macaques and hypothesized that among a broad set of fitness-maximizing strategies, grooming can be used by individuals to enhance social relationships through reciprocity and/or through the interchange of grooming for a different but equivalent service. Overall, they found that social rank played an important role in defining female and/or male social interaction and partner choice in Tibetan macaques. Among female or male intrasexual dyads, the authors found that there

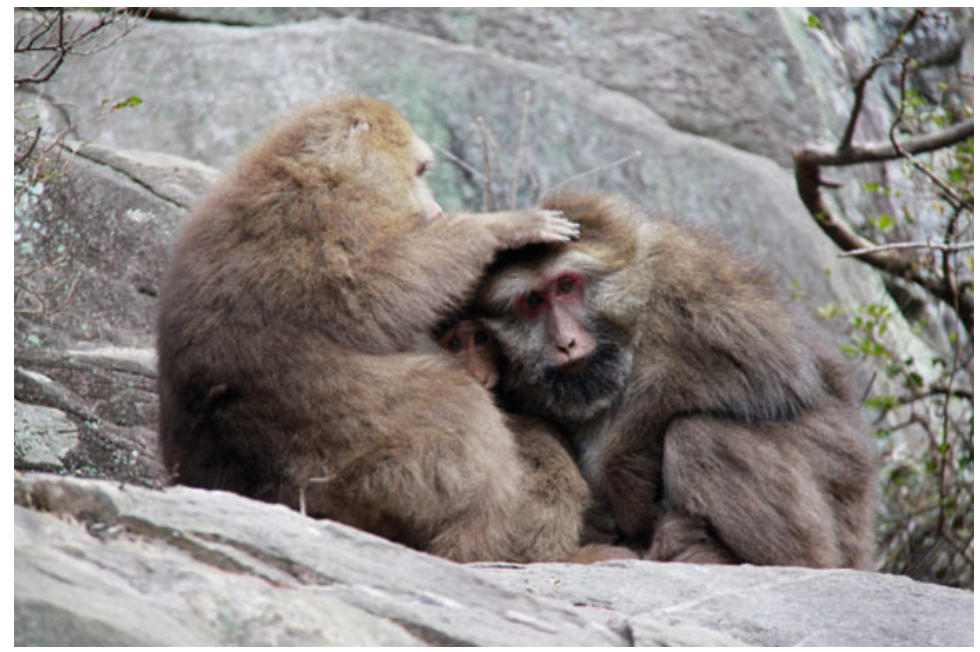

Fig. 4.2 Female-to-female social grooming in Tibetan macaques 
were positive correlations between the average frequency of grooming bouts received (A groom B) and reciprocated (B groom A). These patterns were consistent during the mating and non-mating seasons. Similarly, grooming effort or the time the initiator invested in grooming the recipient was positively correlated with grooming received among female and male intrasexual dyads. Furthermore, the longer the initiator groomed her partner (A groom B), the longer her partner was likely to groom her in return (B groom A). This pattern also was consistent during mating and non-mating periods.

In addition, as indicated in Fig. 4.3, in intrasexual dyads composed of individuals of equal ranks, grooming was reciprocally exchanged (equal rates and equal duration) during both the mating and non-mating seasons. This suggests that, within each sex, dyads composed of animals of equal ranks use reciprocal exchange grooming as a social tool to maintain long-term alliances and partner preferences. Grooming frequency and grooming duration were equal within female-female and male-male dyads consisting of high- and high-ranking individuals, middle- and middle-ranking individuals, and low- and low-ranking individuals during both mating and non-mating periods.

The findings that closely ranked females, regardless of their position in the hierarchy, acted as reciprocal traders are similar to the pattern reported in chacma baboons (P. c. ursinus, Barrett et al. 1999), Japanese macaques (M. fuscata, Ventura et al. 2006), and hamadryas baboons (P. hamadryas hamadryas, Leinfelder et al. 2001). Furthermore, the authors also documented a pattern in which the initiator (A) of a bout invested less and received greater benefit than the recipient. Thus, it appears that among female and/or male Tibetan macaques, the act of initiating a grooming bout was a behavioral investment or strategy to obtain a grooming reward or other social dividend. This effect was strongest when the initiator was higher in rank than the receiver.

\subsection{Exchange Between Different Behaviors}

\subsubsection{Grooming for Tolerance}

Grooming represents one of the most common commodities traded among primates and has long been considered a reliable indicator of both long-term and short-term social tolerance (Dunbar 2010; Schino and Aureli 2010). Seyfarth (1977) has proposed that there exists a balance between a group member's preference for particular grooming partners and competition among females for access to the most valuable grooming partners. According to Seyfarth's model, lower-ranked individuals should compete to groom higher-ranked individuals, who can offer them more effective aid in competitive interactions with other group members. This is expected to result in higher-ranked individuals receiving more grooming than lower-ranked individuals and grooming partners tending to be of similar or adjacent ranks (Henzi et al. 2003). However, Henzi and colleagues (2003) found no 

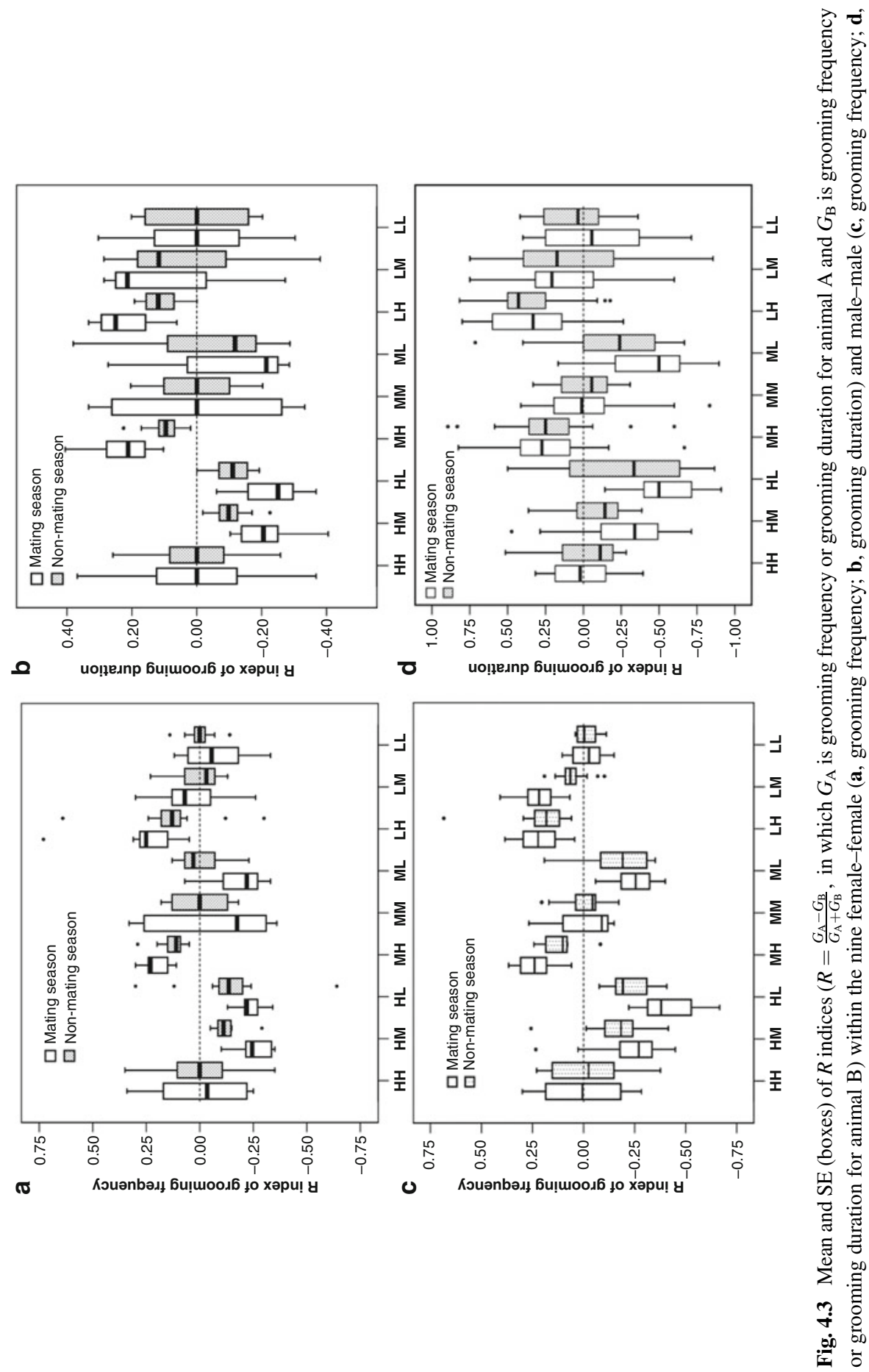


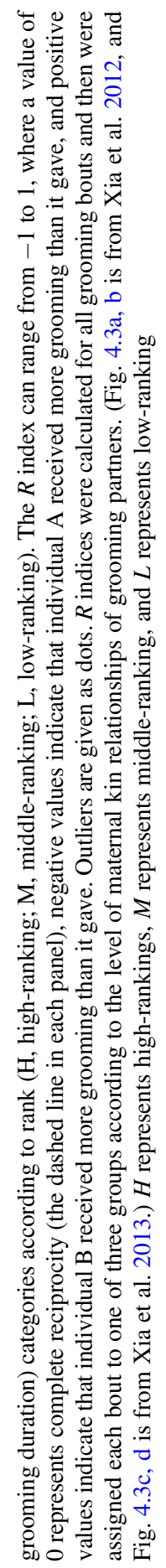


support for Seyfarth's model in their study of chacma baboons. Rather, they argued that in these primates "grooming is unimpeded by restrictions on access to partners" and proposed that baboon females exchanged grooming for increased social tolerance.

In Tibetan macaque society, Xia et al. $(2012,2013)$ also found that in intrasexual dyads composed of individuals of different social ranks (e.g., high- and middleranking females/males, high- and low-ranking females/males, and middle- and low-ranking females/males), initiators received grooming more frequently and for a longer duration than they gave during both mating and non-mating periods. Conversely, in dyads consisting of middle- and high-ranking females, low- and high-ranking females, and low- and middle-ranking females, initiators received grooming less frequently and for a shorter duration than they gave during both mating and non-mating periods. The same results were found among male intrasexual dyads consisting of middle- and high-rankings, low- and higherrankings, and middle- and high-rankings (see Fig. 4.3).

Xia et al. $(2012,2013)$ also found that the effect of grooming reciprocity among female and/or male intrasexual dyads was stronger in the non-mating season. This suggests that during periods of reduced social tension (such as the non-mating period), adult female and/or male Tibetan macaques used grooming to maintain and enhance affiliative social relationships. In contrast, during periods when the rate and the threat of male intrasexual aggression increased (such as the mating season), there was a tendency to promote social affiliation by increasing the duration of grooming. The authors found negative relationships between grooming given and aggression received both among male intrasexual dyads (e.g., as showed in Fig. 4.4) and among female intrasexual dyads. This provided supportive evidence that both female and male Tibetan macaques vary their grooming strategies based on the current rates of within-group intrasexual aggression. The results indicated that highranking females and/or males who received more grooming from lower-ranking males were less aggressive toward their grooming partners. In other words, lowerranking females and/or males who devoted more time to grooming higher-ranking individuals received less aggression than did similarly ranked individuals who groomed higher-ranking females and/or males less frequently. Thus, there is evidence that, for female and/or male intrasexual dyads, Tibetan macaques interchanged grooming for social tolerance and possibly competed for access to partners of higher social rank.

Taken together, Xia et al. $(2012,2013)$ suggest that Tibetan macaques employed an alternative set of social strategies depending on rank relationships. This result highlights the importance of both grooming reciprocal exchange and interchange for tolerance as behavioral mechanisms that regulate female and/or male primate social relationships. 


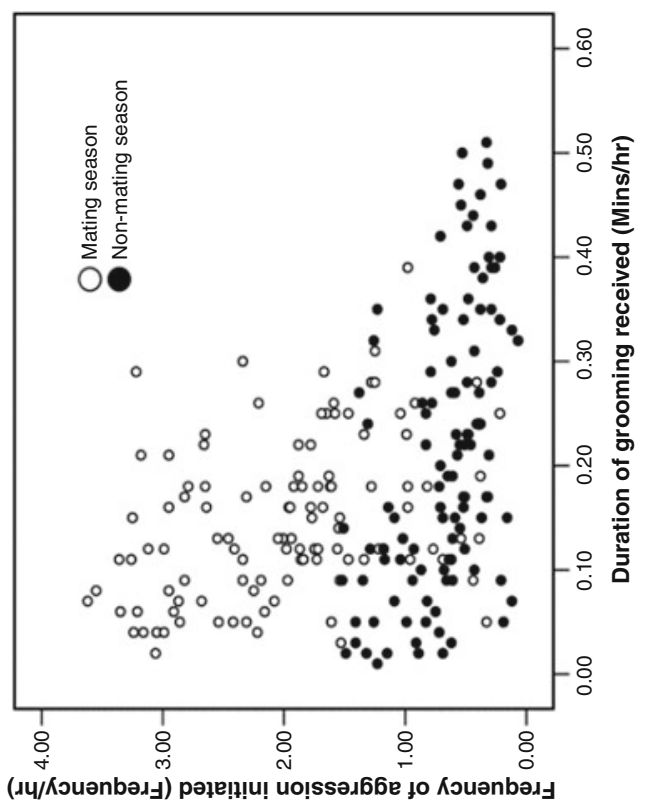

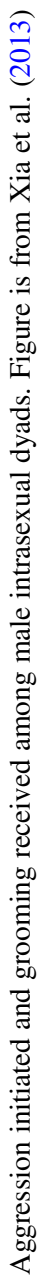

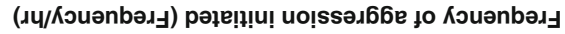




\subsubsection{Grooming for Infant Handling}

Infants are important components in a primate social group, and alloparental care is widespread among primates to facilitate the survival of newborn infants and increase benefits to caregivers (Maestripieri 1994). While females' attraction to infants represents a common feature of primate species, maternal response to infant handling shows a certain degree of variability (Nicolson 1987; Maestripieri 1994). In some species, such as langurs (Presbytis pileata) and vervet monkeys (C. aethiops sabaeus), mothers allow other group members to frequently hold and carry their newborn infants for long durations (Stanford 1992; Fairbanks 1990). In other species, such as baboons and macaques, although mothers are more restrictive of their infants (Nicolson 1987; Altmann 2002), group members still have opportunities to contact newborn infants (see Maestripieri 1994). It has been well documented that infants can be social tools to buffer agonistic behavior from higher-ranked individuals and facilitate dyadic social bonds (Silk and Samuels 1984 in bonnet macaques, M. radiata; Ogawa 1995 in Tibetan macaques). Grooming for females is an effective way to increase the opportunity for accessing Sapajus nigritus infants. For example, in tufted capuchin monkeys (Sapajus apella nigritus), Tiddi et al. (2010) proposed that potential handlers have strongly attracted to infants and grooming their mothers. Similar results have also been found in baboons, P. anubis (Frank and Silk 2009), long-tailed macaques (Gumert 2007b), and golden snub-nosed monkeys, Rhinopithecus roxellana (Wei et al. 2013). These studies imply that grooming might be interchanged for infant handling or vice versa to facilitate dyadic social bonds among females.

In Tibetan macaques, Jiang et al. (2019) found that there were bidirectional relationships between grooming and infant handling among females. Jiang and her colleagues found that there were five patterns of infant handling, including hand touching, mouth licking with teeth chattering, holding, grooming, and bridging. Female Tibetan macaques received more grooming time after giving birth than before parturition, and lactating females invested less grooming time than females without infants. For example, the duration of mothers grooming non-mothers before birth was significantly longer than after birth, whereas the duration of non-mothers grooming mothers after birth was significantly longer than that before birth. Apparently, females with infants are more attractive as grooming partners than females without infants. In a Tibetan macaque social group, adult females with infants received more grooming when this adult female allowed their newborn infants to be handled. For example, the duration of non-mother to mother post-handlinggrooming was higher than the baseline values of daily grooming (see left side of Fig. 4.4) and the grooming duration when these two females were in proximity (see left side of Fig. 4.5). Moreover, with the increase in the number of infants, the amount of grooming received from females without infants decreased.

Additionally, in Tibetan macaques, the frequency of non-mother post-groominginfant handling was higher than the baseline values of daily infant handling (see right side of Fig. 4.4) and the infant handling when these two females are in proximity (see 

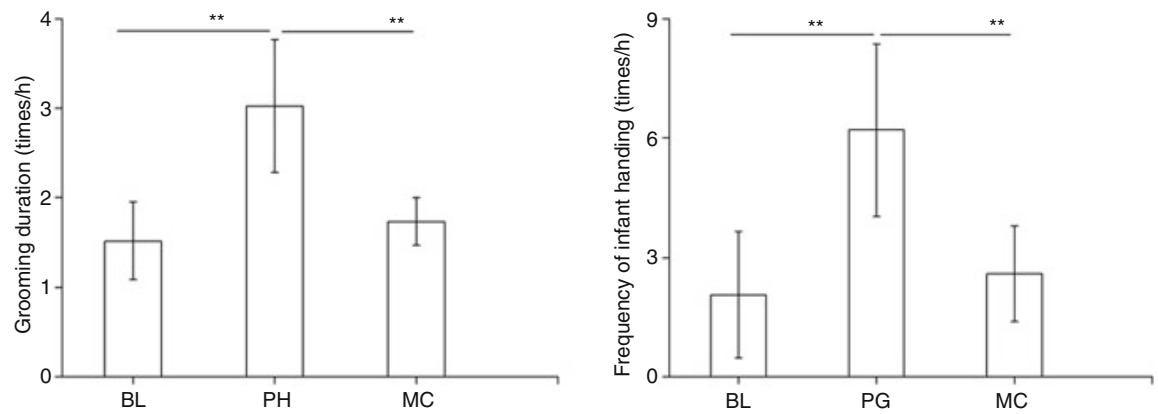

Fig. 4.5 Variation of grooming duration from non-mothers to mothers and frequency of non-mother infant handling. Baseline represents the daily activity, which is from focal sampling data. PH represents post-infant handling-grooming. PG represents post-grooming-infant handling. $\mathrm{MC}$ represents the mean from the data where mothers and non-mothers were in proximity. Figure is modified from Jiang et al. (2019)

right side of Fig. 4.5). These results suggested that females obtained more opportunities for infant handling when she groomed more females with infants; thus, grooming was an effective way to gain access to an infant.

\subsubsection{Agonistic Support for Copulation}

In multi-male-multi-female primates, mating is associated with individual's social rank, with a higher frequency for higher-ranked individuals and lower frequency for lower-ranked individuals (reviewed in Li 1999). Mating is also associated with dyadic affiliative relationships (such as grooming). For example, in a study of Barbary macaques (M. sylvanus), Sonnweber et al. (2015) demonstrated that males initiated grooming after copulations with ejaculation in order to keep females from mating with other males. Agonistic support is predicted to increase mating opportunity. Agonistic support has been defined as instances in which an individual joins an ongoing agonistic interaction and supports one animal by directing aggression against its opponent (Seyfarth and Cheney 1984; Hemelrijk 1994), including intrasexual and intersexual agonistic support.

Wang et al. (2013) used a 15-min focal sampling method to collect data on copulations and agonistic support as a random variable in two groups (YA1 and YA2) of Tibetan macaques in Huangshan, China. A total of 216 male-to-female agonistic support events were collected (YA1 32 and YA2 64 in the non-mating season; YA1 43 and YA2 77 in the mating season). Correlation tests showed that male-to-female agonistic support was correlated with the frequency with which the male copulated with that female in both the mating season and the non-mating season. This indicated that male-to-female agonistic support could increase the male mating opportunities with the female he supported in agonistic events. 

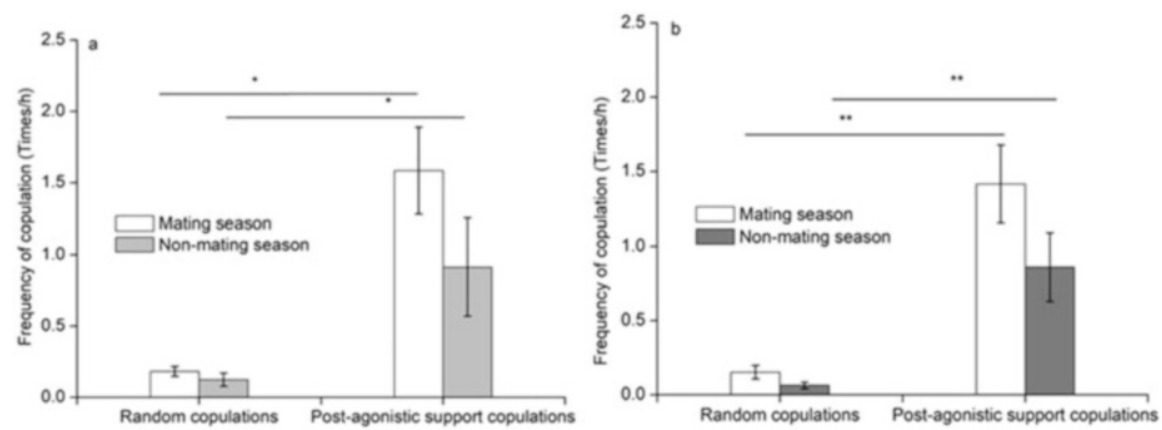

Fig. 4.6 Post-agonistic copulations and post-copulation agonistic supports among female Tibetan macaques. (a) from YA1 group. (b) from YA2 group. $* P<0.05 . * * P<0.01$. Figures are modified from Wang et al. (2013)

In addition, Wang et al. (2013) used a 15-min post-agonistic support focal sampling method to collect copulation data to compare with random values (the average value of copulation from the focal sampling methods). The results showed that, in the YA1 group, copulations in the post-agonistic support observation period (PO) were more frequent, but not significantly so, than random observations (RO) in the non-mating season, and post-agonistic support copulations were significantly more frequent than random observations in the mating season (see Fig. 4.6a). For the YA2 group, copulations in the post-agonistic support period were more frequent than in random observations in both mating and non-mating seasons (see Fig. 4.6b). Taken together, these results indicate that, in Tibetan macaques, male-to-female agonistic support could be interchanged for mating opportunities with females who are participating in an ongoing aggressive event.

Wang et al.'s (2013) study characterized not only the pattern of behavioral exchange in Tibetan macaque social groups but also offers new evidence for how certain mating strategies can increase mating opportunities in males. Male Tibetan macaques copulate under three different circumstances: opportunistic mating, which involves frequent copulation with many different females in the group setting (Li et al. 2015); possessive mating, which involves a single male's attempts to monopolize copulations in spite of the presence of other males ( $\mathrm{Li}$ et al. 2015); and consortships, in which mating takes place between one male and one female, who travel apart from the rest of the group for several hours or days (Li 1999). Xia et al. (in preparation) found that males preferentially mate with their female grooming partners based on dyadic relationships. This indicates that, similar to the findings in studies in Barbary macaques (Sonnweber et al. 2015) and rhesus macaques, $M$. mulatta (Manson 1992), dyadic social relationships play a vital role in obtaining mating opportunities. Agonistic support is one of the effective interactions for males to form and maintain social relationships with females and to obtain and improve copulatory success. In Tibetan macaques, the relationships between agonistic support and copulatory behavior provide insight into understanding malemale competition and female mate choice in social primates. 


\subsection{Conclusions}

In a Tibetan macaque social group, there are diverse exchange networks consisting of different kinds of behavioral exchanges (see Fig. 4.7). Tibetan macaques provide behavioral services for the same behavioral services in return, such as grooming in exchange for grooming. Among intra-sexual dyads (female-female and male-male dyads), grooming investment is exchanged for itself with equal value, in terms of frequency and/or duration. Secondly, Tibetan macaques can also provide behavioral services that are exchanged for different behavioral services of the same category, such as grooming for tolerance. Here, grooming and tolerance are different behaviors; however, both are friendly interactions for facilitating social relationships. Both male and female Tibetan macaques groom group members of the same sex in exchange for tolerance by decreasing aggressive interactions from higher-ranking individuals. In addition, Tibetan macaques provide behavioral services by interchanging different behavioral services with different categories, such as agonistic support for copulations.

In addition, group members can choose their partners for exchanging different types of behavioral services based on their own social status within a group. For example, grooming is regularly traded reciprocally (for grooming) among female and male intrasexual social dyads consisting of similar social ranks. Grooming can be interchanged for rank-related benefits from higher-ranking individuals, such as for tolerance from. Grooming can also be interchanged for opportunities to access newborn infants to facilitate social relationships with the infants' mothers.
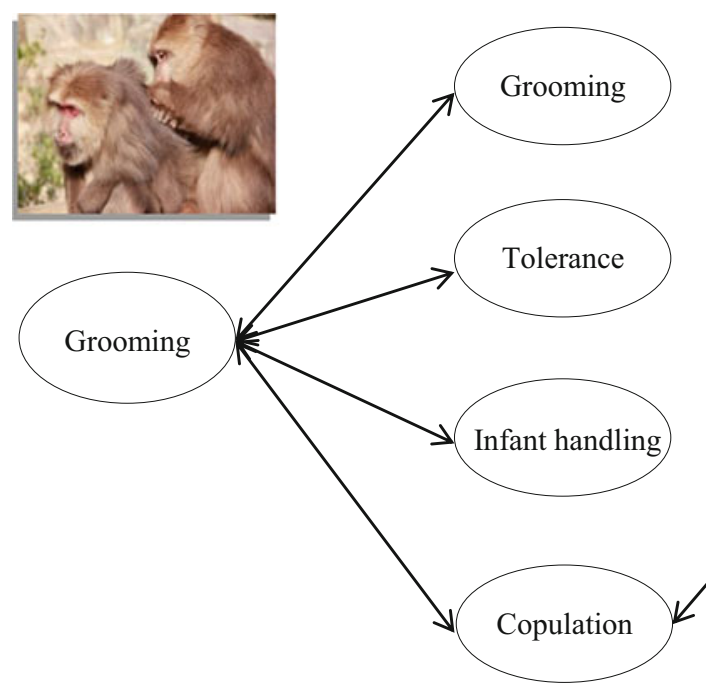

Fig. 4.7 Exchange networks in Tibetan macaque society
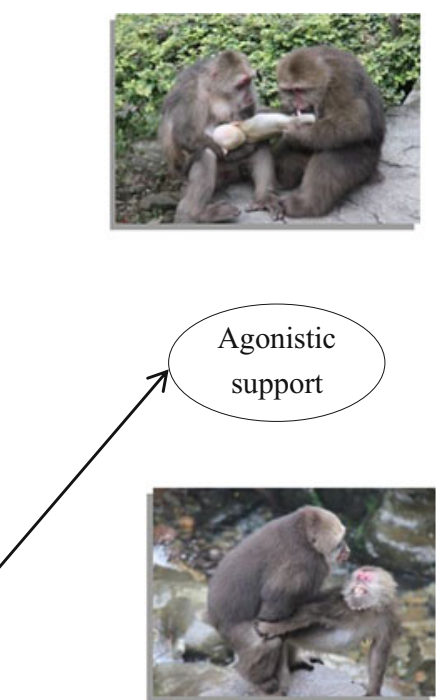
Finally, behavioral exchanges facilitate the establishment and maintenance of social relationships and group stability in multiple ways, by obtaining multiple behavioral services in return. For example, grooming exchanges enhance malemale and female-female social bonds. Grooming with higher-ranking group members allows lower-ranking animals to coexist peacefully with more dominant animals. Non-mothers that groom females with infants could increase their opportunity for accessing infants, and accessing newborn infants could facilitate social relationships between the females who join in infant handling. Among intersexual dyads, male Tibetan macaques support certain females against attacks by other animals and subsequently mate more frequently with that female. This implies that males can interchange copulatory behavior by supporting females to improve mating opportunity.

Although we have several case studies of behavior networks in Tibetan macaque society, we need more evidence to draw a clearer picture about behavioral exchange and interchange in this primate species. Apparently, both males and females may establish diverse forms of behavioral exchange or interchange networks with multiple group members. However, we know little about strategies for partner control or partner choice based on more complex measures of individual and social status (such as personality and dominance) to strengthen social bonds, obtain behavioral services, and maximize fitness. Future studies will need to pay closer attention to the variation of behavioral strategies in connection with group dynamics to better understand how behavioral exchange and interchange are related to the maintenance and stability of social bonds and group networks.

Acknowledgments We are very grateful to the Huangshan Garden Forest Bureau for their permission and support of this work. We also gratefully acknowledge Mr. H.B. Cheng's family for their outstanding logistic support to our study. PAG wishes to thank Chrissie, Sara, and Jenni for their support. DPX wishes to thank Randall C. Kyes, professor at the University of Washington, whom DPX worked with as a visiting scholar, Lori K. Sheeran for her encouragement and support, and Lixing Sun and Rose Amrhein for their help. This work was supported in part by grants from the National Natural Science Foundation of China (No. 31772475; 31672307; 31401981) and the China Scholarship Council.

\section{References}

Altmann J (2002) Baboon mothers and infants. Afr J Ecol 40(4):419-421

Barash DB (1977) Sociobiology and behaviour. Heinemann, London

Barrett L, Henzi SP (2006) Monkeys, markets and minds: biological markets and primate sociality. In: Kappeler PM, van Schaik CP (eds) Cooperation in primates and humans: mechanisms and evolutions. Springer, New York, pp 209-232

Barrett L, Henzi SP, Weingrill T, Lycett JE, Hill RA (1999) Market forces predict grooming reciprocity in female baboons. Proc R Soc Lond B 266:665-670

de Waal FBM (1997) The Chimpanzee's service economy: food for grooming. Evol Hum Behav 18(6):375-386 
Dunbar RIM (2010) The social role of touch in humans and primates: behavioural function and neurobiological mechanisms. Neurosci Biobehav Rev 34(2):260-268

Fairbanks LA (1990) Reciprocal benefits of allomothering for female vervet monkeys. Anim Behav 9:425-441

Frank RE, Silk JB (2009) Impatient traders or contingent reciprocators? Evidence for the extended time-course of grooming exchanges in baboons. Behaviour 146:23-1135

Fruteau C, van de Waal E, van Damme E, Noë R (2011) Infant access and handling in sooty mangabeys and vervet monkeys. Anim Behav 81(1):153-161

Gomes CM, Boesch C (2009) Wild chimpanzees exchange meat for sex on a long-term basis. PLoS One 4:e5116

Gumert MD (2007a) Payment for sex in a macaque mating market. Anim Behav 74(6):1655-1667

Gumert MD (2007b) Grooming and infant handling interchange in Macaca fascicularis: the relationship between infant supply and grooming payment. Int J Primatol 28(5):1059-1074

Gumert MD, Ho MR (2008) The trade balance of grooming and its coordination of reciprocation and tolerance in Indonesian long-tailed macaques (Macaca fascicularis). Primates 49(3):176185

Hemelrijk CK (1994) Support for being groomed in long-tailed macaques, Macaca fascicularis. Anim Behav 48:479-481

Henzi SP, Barrett L, Gaynor D, Greeff J, Weingrill T, Hill RA (2003) Effect of resource competition on the long-term allocation of grooming by female baboons: evaluating Seyfarth's model. Anim Behav 66(5):931-938

Hinde RA (1976) Interactions, relationships and social-structure. Man 11:1-17

Jiang Q, Xia DP, Wang X, Zhang D, Sun BH, Li JH (2019) Interchange between grooming and infant handling in female Tibetan macaques (Macaca thibetana). Zool Res 40:139-145

Kaburu SSK, Newton-Fisher NE (2015a) Egalitarian despots: hierarchy steepness, reciprocity and the grooming-trading model in wild chimpanzees, Pan troglodytes. Anim Behav 99:61-71

Kaburu SSK, Newton-Fisher NE (2015b) Trading or coercion? Variation in male mating strategies between two communities of East African chimpanzees. Behav Ecol Sociobiol 69:1039-1052

Krause J, Ruxton G (2002) Living in groups. Oxford University Press, Oxford

Krebs JR, Davies NB (1984) Behavioral ecology: an evolutionary approach. Blackwell Scientific, Oxford

Leinfelder I, de Vries H, Deleu R, Nelissen M (2001) Rank and grooming reciprocity among females in a mixed-sex group of captive hamadryas baboons. Am J Primatol 55(1):25-42

Li JH (1999) The Tibetan macaque society: a field study. Anhui University Press, Hefei. (In Chinese)

Li ZP, Li JH, Xia DP, Zhu Y, Wang X, Zhang D (2015) Mating strategies of subordinate males in Tibetan macaqus (Macaca thibetana) at Mt. Huangshan, China. Acta Theriologica Sinica 35 (1):29-39. (In Chinese)

Maestripieri D (1994) Social structure, infant-handling, and mother styles in group-living Old World monkeys. Int J Primatol 15:531-553

Manson JH (1992) Measuring female mate choice in Cayo Santiago rhesus macaques. Animl Behav 44:405-416

Morse DH (1980) Behavioral mechanisms in ecology. Harvard University Press, Cambridge, MA

Newton-Fisher NE, Lee PC (2013) Grooming reciprocity in wild male chimpanzees. Anim Behav 81(2):439-446

Nicolson N (1987) Infants, mothers, and other females. In: Smuts BB, Cheney DL, Seyfarth RM, Wrangham RM, Struhsaker TT (eds) Primate societies. University of Chicago Press, Chicago, IL, pp 330-342

Noë R (2001) Biological markets: partner choice as the driving force behind the evolution of mutualisms. In: Noe R, van Hooff JARAM, Hammerstein P (eds) Economics in nature: social dilemmas, mate choice and biological markets. Cambridge University Press, Cambridge, pp 93-118 
Noë R (2006) Digging for the roots of trading. In: Kappeler PM, van Schaik CP (eds) Cooperation in primates and humans: mechanisms and evolution. Springer, Berlin, pp 223-251

Noë R, Hammerstein P (1994) Biological market: supply and demand determine the effect of partner choice in cooperation, mutualism and mating. Behav Ecol Sociobiol 35:1-11

Noë R, Hammerstein P (1995) Biological markets. Trends Ecol Evol 10:336-339

Noë R, Voelkl B (2013) Cooperation and biological markets: the power of partner choice. In: Sterelny K, Joyce R, Calcott B, Fraser B (eds) Cooperation and its evolution. MIT Press, Cambridge, MA, pp 131-152

Ogawa H (1995) Bridging behavior and other affiliative interactions among male Tibetan macaques (Macaca thibetana). Int J Primatol 16:707-729

Port M, Clough D, Kappeler PM (2009) Market effects offset the reciprocation of grooming in freeranging redfronted lemurs, Eulemur fulvus rufus. Anim Behav 77(1):29-36

Sánchez-Amaro A, Amici F (2015) Are primates out of the market? Anim Behav 110:51-60

Schino G, Aureli F (2009) Reciprocal altruism in primates: partner choice, cognition, and emotions. Adv Study Behav 39:45-69

Schino G, Aureli F (2010) Primate reciprocity and its cognitive requirements. Evol Anthropol Issues News Rev 19(4):130-135

Schino G, Aureli F (2017) Reciprocity in group-living animals: partner control versus partner choice. Biol Rev 92:665-672

Schino G, Polizzi di Sorrentino EP, Tiddi B (2007) Grooming and coalitions in Japanese macaques (Macaca fuscata): partner choice and the time frame reciprocation. J Comp Psychol 121:181-188

Schino G, di Giuseppe F, Visalberghi E (2009) The time frame of partner choice in the grooming reciprocation of Cebus paella. Ethology 115:70-76

Seyfarth RM (1977) A model of social grooming among adult female monkeys. J Theor Biol 65:671-698

Seyfarth RM, Cheney DL (1984) Grooming, alliances and reciprocal altruism in vervet monkeys. Nature 308:541-542

Silk JB, Samuels A (1984) Triadic interactions among Macaca radiata: passports and buffers. Am J Primatol 6:373-376

Sonnweber RS, Massen JJM, Fitch WT (2015) Post-copulatory grooming: a conditional mating strategy? Behav Ecol Sociobiol 69:1749-1759

Stanford C (1992) The costs and benefits of allomothering in wild capped langurs (Presbytis pileata). Behav Ecol Sociobiol 30:29-34

Sussman RW, Garber PA (2011) Cooperation, collective action, and competition in primate social interactions. In: Campbell CJ, Fuentes A, MacKinno KC, Bearder S, Stumpf R (eds) Primates in perspective, vol 2. Oxford University Press, New York, pp 587-599

Tiddi B, Aureli F, Schino G (2010) Grooming for infant handling in tufted capuchin monkeys: a reappraisal of the primate infant market. Anim Behav 79:1115-1123

van Schaik CP (1989) The ecology of social relationships amongst female primates. In: Standen V, Foley RA (eds) Comparative socioecology: the behavioural ecology of humans and other animals. Blackwell Scientific, Oxford, pp 195-218

Ventura R, Majolo B, Koyama NF, Hardie S, Schino G (2006) Reciprocation and interchange in wild Japanese macaques: grooming, cofeeding, and agonistic support. Am J Primatol 68 (12):1138-1149

Wang S, Li JH, Xia DP, Zhu Y, Sun BH, Wang X, Zhu L (2013) Male-to-female agonistic support for copulation in Tibetan macaques (Macaca thibetana) at Huangshan, China. Zool Res 34:139-144

Wei W, Qi XG, Garber PA, Guo ST, Zhang P, Li BG (2013) Supply and demand determine the market value of access to infants in the golden snub-nosed monkey (Rhinopithecus roxellana). PLoS One 8(6):e65962

Wilson EO (1975) Sociobiology. Harvard University Press, Cambridge, MA 
Wrangham RW (1980) An ecological model of female-bonded primate groups. Behaviour 75:262-299

Xia DP, Li JH, Garber PA, Sun L, Zhu Y, Sun BH (2012) Grooming reciprocity in female Tibetan macaques Macaca thibetana. Am J Primatol 74:569-579

Xia DP, Li JH, Garber PA, Matheson MD, Sun BH, Zhu Y (2013) Grooming reciprocity in male Tibetan macaques. Am J Primatol 75:1009-1020

Open Access This chapter is licensed under the terms of the Creative Commons Attribution 4.0 International License (http://creativecommons.org/licenses/by/4.0/), which permits use, sharing, adaptation, distribution and reproduction in any medium or format, as long as you give appropriate credit to the original author(s) and the source, provide a link to the Creative Commons licence and indicate if changes were made.

The images or other third party material in this chapter are included in the chapter's Creative Commons licence, unless indicated otherwise in a credit line to the material. If material is not included in the chapter's Creative Commons licence and your intended use is not permitted by statutory regulation or exceeds the permitted use, you will need to obtain permission directly from the copyright holder. 